Arq. Bras. Med. Vet. Zootec., v.68, n.2, p.448-456, 2016

\title{
Modelos de regressão aleatória para características de crescimento de bovinos da raça Nelore do estado de Mato Groso
}

\author{
[Random regression models to traits of growth in the Nellore breed in Mato Grosso state] \\ C.V. Araújo ${ }^{1}$, W.F. Nehls ${ }^{1}$, M.M.M. Laureano ${ }^{1}$, R. Zubler ${ }^{1}$, R.B. Lôbo ${ }^{2}$, L.G.G. Figueiredo ${ }^{2}$, \\ S.I. Araújo ${ }^{1}$, L.A.F. Bezerra ${ }^{2}$ \\ ${ }^{1}$ Instituto de Ciências Agrárias e Ambientais - Universidade Federal de Mato Grosso - MT \\ ${ }^{2}$ Associação Nacional de Criadores e Pesquisadores - Ribeirão Preto, SP
}

\begin{abstract}
RESUMO
Foram utilizados 138.976 registros de informações de pesos corporais variando de 60 a 610 dias de idade, provenientes de 27.327 animais da raça Nelore, oriundos de rebanhos do estado do Mato Grosso, com o objetivo de descrever a variabilidade genética e estimar parâmetros genéticos para o peso corporal em diferentes idades, utilizando-se modelos de regressão aleatória. O modelo empregado incluiu efeitos fixos de grupo de contemporâneos e idade da vaca ao parto como covariáveis, além de efeitos aleatórios genético aditivo direto, genético materno, ambiente permanente de animal, ambiente permanente materno e efeito de ambiente temporário. O modelo de regressão aleatória mais adequado foi o que empregou função de covariância com polinômios de quarta ordem para descrição da variabilidade de todos os efeitos e duas classes de variância residual. As estimativas de variância genética aditiva direta e de ambiente permanente de animal aumentaram com a idade dos animais. As variâncias genética materna e de ambiente permanente materno exibiram comportamento semelhante, com maiores valores na fase de aleitamento. Os coeficientes de herdabilidade estimados variam de 0,25 a 0,43 , com maiores valores nas idades mais avançadas na trajetória de crescimento dos animais. Esses resultados indicaram presença de variabilidade genética suficiente para obtenção de ganho genético expressivo por meio da seleção, principalmente após desmama. Os resultados encontrados para a correlação genética aditiva direta exibiram baixas correlações entre pesos nas idades iniciais e finais, porém pesos altamente correlacionados entre idades mais próximas. As correlações genéticas estimadas entre os pesos da desmama com os pesos até 610 dias de idade foram altas e positivas e indicam que os genes responsáveis por maiores pesos nesse período, em sua maioria, são os mesmos.
\end{abstract}

Palavras-chave: correlação genética, gado de corte, herdabilidade, pesos, polinômios ortogonais de Legendre

\begin{abstract}
ABSTRAC
In this study 138,976 records of live weight between 60 to 610 days of age, from 27,327 Nellore cattle breed, from herds in Mato Grosso State were used in order to describe the genetic variability and to estimate genetic parameters for the live weight at different ages, using random regression models. The model included the fixed effects of contemporary group and age of cow at calving as covariate, random effects of direct additive genetic, maternal genetic, animal and maternal permanent environmental and temporary environment effect. The most appropriate random regression model employed the covariance function with fourth order polynomials to describe the variability of all effects and two residual variance classes. Estimates of direct additive genetic variance and animal permanent environment increased with the age of the animals. Maternal genetic variances and maternal permanent environment exhibited similar behavior, with higher values in pre weaning. The estimated heritability coefficients ranged from 0.25 to 0.43, with higher values at older ages in the growth trajectory of the animals. These results showed the presence of sufficient genetic variability to obtain significant genetic gain through selection,
\end{abstract}

Recebido em 4 de abril de 2015

Aceito em 15 de novembro de 2015

E-mail : araujocv@bol.com.br 
especially after weaning. The results for the direct additive genetic correlation exhibited low correlations between weights in initial and final ages, however, highly correlated weights between nearest ages. Genetic correlation estimates between weaning with weights up to 610 days of age were high and positive and indicate that most of the genes responsible for higher weights in this period are the same.

Keywords: genetic correlation, beef cattle, heritability, weights, orthogonal polynomials of legendre

\section{INTRODUÇ̃̃O}

Para variáveis que apresentam variação contínua no tempo ou no espaço, tem sido observada a crescente utilização dos modelos de regressão aleatória (MRA). Entre as vantagens da metodologia de MRA, reside o fato de que os parâmetros de uma característica quantitativa sejam descritos como uma função sob uma escala contínua, ocasionando mudança no panorama descontínuo das estimativas de variâncias e covariâncias (Kirkpatrick et al., 1990). Outra vantagem do uso desses modelos na descrição do crescimento ponderal de bovinos consiste em considerar a presença de heterogeneidade da variância residual, ajustando diferentes estimativas em diferentes períodos ao longo da trajetória de crescimento dos animais, permitindo maior precisão nas estimativas de parâmetros genéticos com maior acurácia nas predições de valores genéticos.

A aplicação de MRA para características de crescimento em bovinos permite estimar e predizer parâmetros e valores genéticos para qualquer idade desejada, mesmo para idades em que o animal não tenha sido mensurado. Com a utilização desses modelos, não há necessidade de se criarem classes de idades ou de se utilizarem fatores de ajuste para idade, uma vez que esta está incluída na matriz de delineamento. Além disso, os MRA permitem utilização eficiente de toda a informação disponível de cada animal, já que todas as medidas do animal e de seus parentes são utilizadas para a avaliação genética, com potencial aumento da acurácia das estimativas (Meyer, 2005).

No melhoramento genético animal, os MRA vêm sendo empregados na estimação de parâmetros genéticos de diversas características em gado de corte (Arango et al., 2004; Nephawe, 2004; Nishida et al., 2006; Boligon, 2008) e na descrição de curvas de crescimento (Meyer, 2005; Dias et al., 2006).
Assim, objetivou-se com este estudo identificar quais os graus de polinômios de Legendre a serem utilizados nas funções de covariâncias para descrição das variâncias dos efeitos aleatórios pertencentes ao MRA para descrever a variação da trajetória de crescimento dos animais da raça Nelore, bem como utilizá-la na estimação de parâmetros genéticos. Além disso, buscou-se avaliar e corrigir a presença de heterogeneidade de variâncias residuais ao longo da trajetória de crescimento dos animais e estimar parâmetros genéticos para o peso corporal dos animais em diferentes idades ao longo da trajetória de crescimento dos animais.

\section{MATERIAL E MÉTODOS}

Foram utilizados 138.976 registros de informações de pesos corporais variando de $60 \mathrm{a}$ 610 dias de idade, provenientes de 27.327 animais da raça Nelore, proles de 467 reprodutores acasalados com 16.937 matrizes, oriundas de 14 rebanhos do estado de Mato Grosso, participantes do Programa de Melhoramento Genético da Raça Nelore Nelore Brasil da Associação Nacional de Criadores e Pesquisadores.

Os meses de nascimento foram agrupados em duas estações: estação da seca, que corresponde aos de menor incidência de chuvas (de abril a setembro), e estação das águas, que compreende os de maior incidência de chuvas (de outubro a março). Foram gerados 551 níveis de grupos de contemporâneos, contemplando-se efeitos fixos de rebanho, ano e estação de nascimento dos animais e sexo do animal (74.976 registros de machos e 64.000 registros de fêmeas).

A idade da vaca ao parto, com média de $87,54 \pm 36,01$ meses, variando de 23 a 195 meses, foi utilizada como covariável, ajustando-se os efeitos linear e quadrático.

O modelo de regressão aleatória utilizado para obtenção das soluções fixas e aleatórias adotou a 
função polinomial de Legendre com diferentes graus, representado como:

$y=X b+Z_{1} \alpha+Z_{2} \gamma+W_{1} \delta+W_{2} p+\varepsilon$, em que y é o vetor de observações referentes ao peso corporal do animal em diferentes idades; $\mathbf{b}$ é o vetor de efeitos fixos, incluindo o efeito de grupo de contemporâneos e os efeitos linear e quadrático da idade da vaca ao parto como covariáveis; $\alpha$ é o vetor aleatório dos coeficientes de regressão genéticos aditivos diretos; $\gamma$ é o vetor aleatório dos coeficientes de regressão genéticos maternos; $\delta$ é o vetor aleatório dos coeficientes de regressão de ambiente permanente do animal; p é o vetor aleatório dos coeficientes de regressão de ambiente permanente materno; $\mathrm{X}, \mathrm{Z}_{1}, \mathrm{Z}_{2}, \mathrm{~W}_{1}, \mathrm{~W}_{2}$ são matrizes de incidência correspondentes a $\mathbf{b}$, $\alpha, \gamma$ e p, respectivamente. As pressuposições associadas ao modelo foram definidas como:

$$
\left[\begin{array}{c}
a \\
\gamma \\
\delta \\
p \\
\varepsilon
\end{array}\right] \sim N(0, V) ; V=\left[\begin{array}{c}
K_{A} \otimes A \\
0 \\
0 \\
0 \\
0
\end{array}\right.
$$

$\begin{array}{cc}0 & 0 \\ K_{M} \otimes A & 0 \\ 0 & K_{C} \otimes I_{N a} \\ 0 & 0 \\ 0 & 0\end{array}$

$\left.\begin{array}{cc}0 & 0 \\ 0 & 0 \\ 0 & 0 \\ K_{Q} \otimes I_{N m} & 0 \\ 0 & R\end{array}\right]$,

em que $\mathrm{K}_{\mathrm{A}}, \mathrm{K}_{\mathrm{M}}, \mathrm{K}_{\mathrm{C}}, \mathrm{K}_{\mathrm{Q}}$ são matrizes dos coeficientes das funções de covariância para os efeitos genético aditivo direto, genético materno, de ambiente permanente de animal e de ambiente permanente materno, respectivamente; A é a matriz de parentesco entre indivíduos; I é uma matriz identidade; $\mathrm{Na}, \mathrm{Nm}$ são os números de animais com registros de peso corporal e número de mães, respectivamente; $\mathrm{R}$ é a matriz diagonal contendo as variâncias residuais.

O grau do polinômio de Legendre utilizado nas funções de covariâncias para descrição da curva média fixa, bem como na descrição dos efeitos aleatórios do modelo, variou de três a seis (funções quadráticas até quinto grau).

Classes de variância residuais foram compostas variando de uma (resíduo homogêneo) até seis (variâncias residuais heterogêneas), dispostas da seguinte forma: de 60 a $120 ; 121$ a $250 ; 251$ a $350 ; 351$ a $450 ; 451$ a 550 e 551 a 610 dias de idade.

A comparação entre os modelos, com diferentes graus de polinômios e diferentes classes residuais, foi realizada por meio dos critérios de informação Akaike (AIC) e bayesiano de Schwarz (BIC), que permitem a comparação de

modelos não aninhados e penalizam modelos mais parametrizados, sendo o BIC o mais rigoroso, ou seja, o critério que favorece modelos mais parcimoniosos (Wolfinger, 1993). Os critérios de informação são definidos como $\mathrm{AIC}=-2 \log (\mathrm{L})+2 \mathrm{p}$ e $\mathrm{BIC}=2 \log (\mathrm{L})+$ $\mathrm{p}(\log (\mathrm{N}-\mathrm{r})$, em que: $p=$ número de parâmetros do modelo; $N=$ total de observações; e $r=$ posto da matriz $X$ (matriz de incidência para os efeitos fixos). Valores menores de AIC e BIC indicam melhor ajuste do modelo.

As funções de covariâncias foram estimadas utilizando-se modelos de regressão aleatória pelo método de máxima verossimilhança restrita, empregando-se o programa estatístico WOMBAT (Meyer, 2006)

\section{RESULTADOS E DISCUSSÃO}

A média de peso corporal dos animais avaliados e o número de registros, de acordo com a idade, estão apresentados na Fig. 1. A média do peso dos animais foi de $230 \pm 76,62 \mathrm{~kg}$, variando de 47 a $538 \mathrm{~kg}$, com idade média de $330,54 \pm 154,50$ dias. O peso aumentou linearmente com a idade, e o número de informações observadas foi menor nos extremos do intervalo avaliado. 


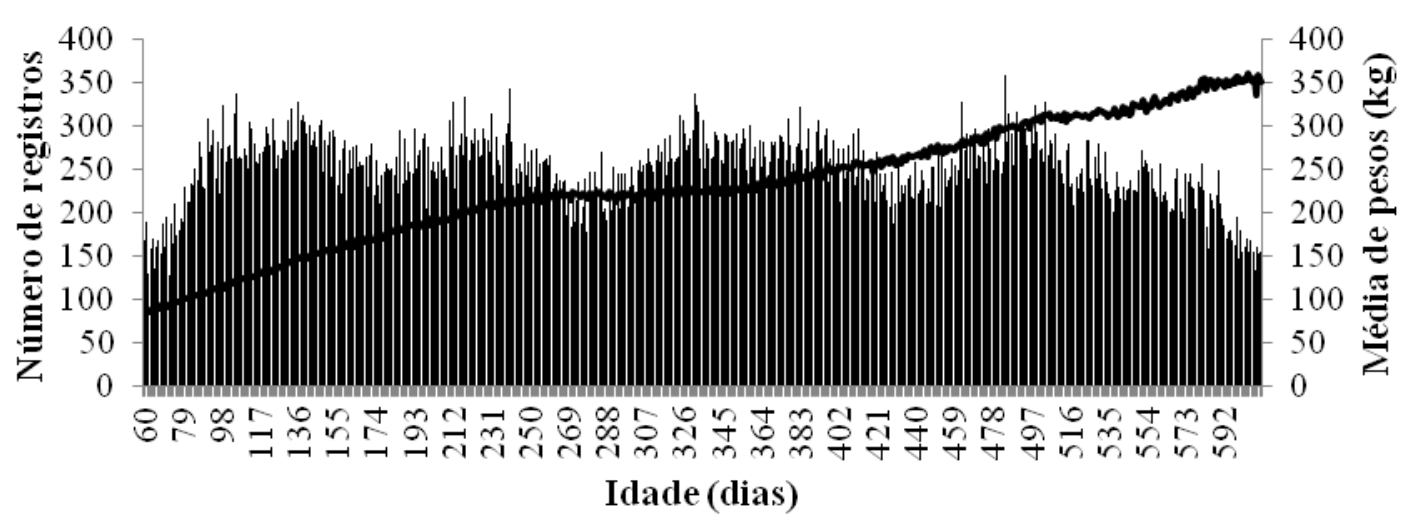

Figura 1. Número de registros (barras) e média de peso, em kg (linha), de animais da raça Nelore no intervalo de 60 a 610 dias de idade

Os valores para os critérios de informação de Akaike (AIC) e bayesiano de Schwarz (BIC) indicaram-no como o modelo mais adequado para descrever as variâncias dos efeitos aleatórios genético aditivo direto, de ambiente permanente do animal e materno, além do efeito genético materno, o que empregou função de covariância com polinômios de quarta ordem (grau cinco) para descrição da variabilidade de todos os efeitos aleatórios genéticos e de ambiente permanente, com duas classes de variância residual, de 60 a 120 e de 121 a 610 dias de idade. Resultado semelhante foi obtido por Santoro et al. (2005), com registros de pesos do nascimento até 720 dias de idade em animais da raça Nelore; por outro lado, Albuquerque e Meyer (2001), Nobre et al. (2003) e Dias et al. (2006) encontraram como mais satisfatórios polinômios cúbicos para modelar a trajetória de crescimento de animais zebuínos. Souza Júnor et al. (2010) elegeram o modelo de regressão aleatória com funções de covariância que utilizaram polinômios de ordens 4, 3, 6, 3 para os efeitos genético aditivo direto e materno, de ambiente permanente de animal e materno, respectivamente. Da mesma forma, Bertipaglia (2013) e Baldi et al. (2010) verificaram que o melhor modelo para o ajuste de dados foi aquele que considerou diferentes graus (de 3 a 6 ) para modelar a variância dos diferentes efeitos aleatórios.

Meyer (2003) relata que o uso de polinômios de alto grau poderia ocasionar dificuldades de convergência e estimativas irreais de variâncias para maiores idades. A mesma autora sugeriu que uma regressão quadrática para todos os efeitos aleatórios seria uma escolha conservadora e sensível. Esse comportamento não foi verificado por Santoro et al. (2005), que observaram pior ajuste para as funções quadráticas, indicado pelos critérios de convergência AIC e BIC.

Arango et al. (2004) e Baldi et al. (2010) apontam dificuldades na aplicação de polinômios ortogonais em dados de crescimento de bovinos, a exemplo de que não modelam adequadamente o padrão cíclico dos dados (típico em características de crescimento submetidas a efeitos estacionais do clima) e, ainda, a falta de comportamento assintótico, já que, em geral, o crescimento animal atinge um "plateau" à maturidade. Além disso, os autores sugerem que o uso de polinômios de alto grau, principalmente para modelar os efeitos de ambiente permanente do animal, além de conduzir ao aumento do número de parâmetros a serem estimados com consequente aumento nas exigências da capacidade computacional, pode gerar erros das estimativas.

Dessa forma, fica claro que não há uma única recomendação sobre o grau do polinômio necessário a ser utilizado no modelo de regressão aleatória, uma vez que a quantidade de informação por data de pesagens pode influenciar na necessidade da quantidade de graus do polinômio que melhor se ajustará aos dados.

De acordo com os valores dos critérios de convergência logaritmo da função de verossimilhança, critério de informação de 


\section{Araújo et al.}

Akaike, critério de informação (AIC) e bayesiano de Schwarz (BIC), ficou evidente a presença heterogeneidade de variância residual sobre o peso corporal dos animais, ao longo da trajetória de crescimento, com a necessidade do ajuste de duas classes de variância residual, discriminando os períodos de aleitamento e pósaleitamento.

Huisman et al. (2002) discutem que assumir homogeneidade de variância significa que $o$ ambiente temporário afeta igualmente todas as idades, o que nem sempre é verdadeiro. Além disso, grande parte da variação residual pode influenciar a variância de ambiente permanente de animal.

A literatura apresenta vários trabalhos que indicam que considerar estruturas heterogêneas de variância residual é mais adequado para ajustar pesos de bovinos ao longo do crescimento (Meyer, 1999; Albuquerque e Meyer, 2001; Nobre et al., 2003; Dias et al., 2006; Boligon et al., 2008; Souza Junior et al., 2010; Bertipaglia, 2013). Por outro lado, Santoro et al. (2005) verificaram melhor ajuste do modelo de regressão aleatória considerando o resíduo homogêneo na descrição da variação do desenvolvimento ponderal em animais Nelore criados no Pernambuco.

Toral et al. (2009) ressalta que o agrupamento de classes adjacentes e que apresentam variâncias semelhantes, desde que não interfira no ajuste dos dados, é uma estratégia bastante interessante para reduzir o número de parâmetros dos modelos, tornando-os mais parcimoniosos. De acordo com os mesmos autores, alterações das variâncias residuais são provenientes do menor número de informações devido à maior dificuldade de obtenção de medidas em animais mais velhos. Além disso, alguns animais podem receber tratamentos diferenciados ao longo da vida sem que esses efeitos sejam corretamente considerados nas análises.

As estimativas dos componentes de variância para o efeito genético aditivo, de ambiente permanente de animal e de vaca e genético materno podem ser observadas na Fig. 2.

As estimativas de variância genética aditiva direta aumentaram com a idade, passando de
$80 \mathrm{~kg}^{2}$ (aos 60 dias) para $1261 \mathrm{~kg}^{2}$ (aos 610 dias de idade). Houve aumento mais acentuado a partir dos 560 dias de idade, provavelmente devido ao menor número de informações, o que pode ter gerado maior variação dos dados. De modo semelhante, aumentos nas estimativas de variância genética aditiva direta durante $\mathrm{o}$ crescimento dos animais foram relatados por Albuquerque e Meyer (2001), Nobre et al. (2003) e Dias et al. (2006) e Souza Junior et al. (2010).

Para o efeito de ambiente permanente de animal, observou-se aumento nas estimativas de acordo com a idade. No intervalo de 100 a 330 dias de idade, essa variância teve o mesmo comportamento da variância genética aditiva e, a partir dessa idade, apresentou crescimento mais acentuado até os 600 dias de idade.

As variâncias genética materna e de ambiente permanente materno exibiram comportamento semelhante, entretanto $o$ efeito permanente materno apresentou estimativas pouco superiores. As estimativas de variância genética materna apresentaram valores crescentes até, aproximadamente, 200 dias de idade, com tendência de queda nas idades mais avançadas, voltando a aumentar no final do período estudado. Resultados semelhantes foram descritos por Albuquerque e Meyer (2001), Nobre et al. (2003) e Dias et al. (2006).

As estimativas de herdabilidade direta e materna, proporções de ambiente permanente de animal e materno em relação à variação total para o peso corporal em idades pertencentes ao intervalo da trajetória estudada são exibidas na Tab.1. O valor médio das estimativas dos coeficientes de herdabilidade em toda a trajetória de crescimento dos animais foi igual a 0,33 , variando de 0,25 a 0,43 .

As estimativas de herdabilidade direta obtidas apresentaram tendência de queda de $60(0,39)$ até 90 dias $(0,25)$ e, posteriormente, aumento nas estimativas, atingindo o valor máximo aos 610 dias de idade $(0,43)$. As altas estimativas observadas no final do período avaliado são devido às maiores variâncias genéticas aditivas. Comportamento semelhante foi verificado por Meyer (2005), Souza Junior et al. (2010) e Bertipaglia (2013). 


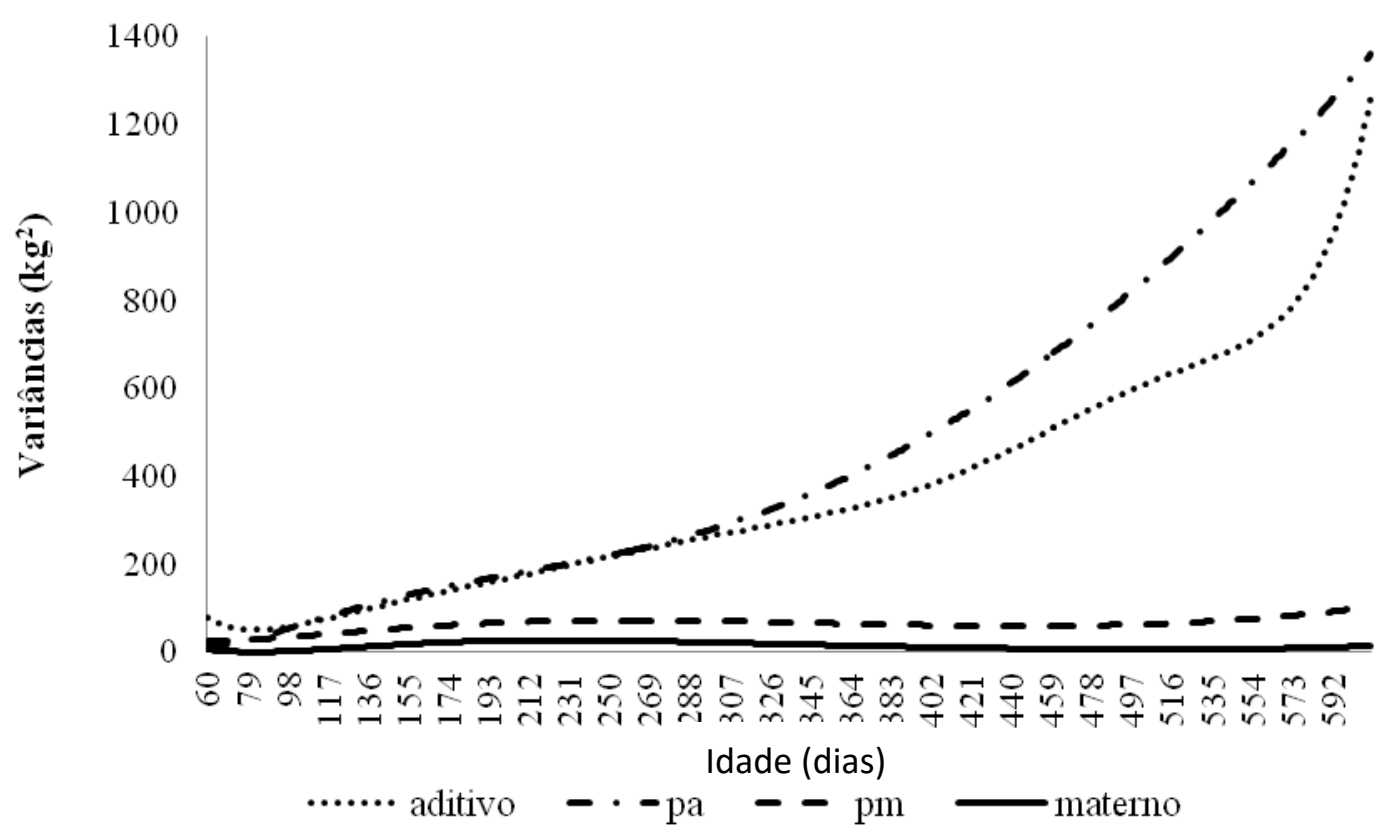

Figura 2. Estimativas dos componentes de variância para o efeito genético aditivo (aditivo), de ambiente permanente de animal (pa) e de vaca (pm) e genético materno (materno) para o desenvolvimento ponderal de animais da raça Nelore.

Albuquerque e Meyer (2001) comentam que o uso de polinômios de alto grau conduz a problemas de inconsistências nas variâncias e covariâncias em idades extremas, em razão da maior ênfase que os polinômios colocam nas observações localizadas nos extremos da curva. Segundo Dias et al. (2006), a partição dos efeitos aleatórios separando os efeitos genéticos e de ambiente em direto e materno é essencial para modelar de maneira adequada as mudanças durante as fases de crescimento. A modelagem do resíduo e a ordem do ajuste dos polinômios podem interferir na estimação dos componentes de variância. Assim, as estimativas de parâmetros genéticos podem ser influenciadas pelo modelo de regressão adotado e pela quantidade de informação de registros fenotípicos disponíveis ao longo da trajetória de crescimento, sendo sempre necessário testar novamente os graus dos polinômios das funções de covariâncias na estimação de componentes de variâncias a cada atualização do banco de dados da população, visto que baixas quantidades de informações de pesos corporais provenientes do início e final da trajetória ocasionam superestimação das estimativas de herdabilidade.

As estimativas obtidas para herdabilidade direta sugerem haver variabilidade genética aditiva necessária e suficiente para a obtenção de ganho genético expressivo sobre o peso corporal dos animais, quando a seleção é realizada com base na informação do próprio indivíduo, principalmente no peso ao sobreano.

Estimativas de herdabilidades com tendência de redução entre o nascimento e a desmama e posterior aumento até o sobreano foram observadas por vários autores (Albuquerque e Meyer, 2001; Meyer, 2003; Nobre et al., 2003 e Dias et al., 2006). Santoro et al. (2005) verificaram estimativas de herdabilidade de baixa à média até aproximadamente 60 dias, sendo alta para as demais idades, ficando entre 0,50 e 0,60 .

As estimativas de componentes de variância das proporções de ambiente permanente de animal $\left(\mathrm{pa}^{2}\right)$ em relação à variação total para o peso corporal apresentaram menor estimativa no início da trajetória, igualando-se aos valores das estimativas de herdabilidade $(0,24)$ por volta do $100^{\circ}$ dia de idade, e, por volta dos 280 dias de idade, as estimativas de $\mathrm{pa}^{2}$ foram maiores que as herdabilidades estimadas; aos 579 dias, atingiram o valor máximo de 0,52 . A média de $\mathrm{pa}^{2}$ foi igual a 0,39 , variando de 0,06 a 0,52 . Os resultados obtidos revelam alta repetibilidade do peso 


\section{Araújo et al.}

corporal com o incremento da idade. De acordo com Bertipaglia (2013), o aumento desta variância no início da vida do animal está relacionado à maior susceptibilidade às variações ambientais nesta fase do desenvolvimento do indivíduo, que tende a ser reduzida com o passar do tempo.

Para as estimativas de componentes de variância das proporções de ambiente materno em relação à variação total para o peso corporal $\left(\mathrm{pm}^{2}\right)$, verificaram-se valores iguais a 0,12 no início da trajetória, atingindo máximo valor de 0,15 aos 90 dias de idade aproximadamente, declinando suavemente até o final da trajetória $(0,03)$; em média, esse parâmetro apresentou o valor de 0,08 em toda a trajetória.

A herdabilidade materna apresentou baixas estimativas ao longo da trajetória, com maiores valores $(0,05)$ ocorrendo, aproximadamente, no intervalo de 180 a 220 dias de idade. A estimativa média da herdabilidade materna em toda trajetória foi igual a 0,02. Embora de baixa magnitude, a tentativa de maior sucesso de resposta à seleção para habilidade materna pode ser esperada se for realizado o processo de seleção baseado em pesos obtidos próximos à desmama, pelo fato de a variância genética materna apresentar seus maiores valores. Resultados semelhantes foram relatados por Albuquerque e Meyer (2001), Dias et al. (2006) e Souza Júnior et al.(2010). É importante ressaltar que a seleção para habilidade materna resulta em matrizes com maior potencial genético para produção de leite, principal componente do efeito materno, e essa característica é antagônica ao desenvolvimento ponderal.

Tabela 1. Estimativa de herdabilidade para o peso corporal em algumas idades, dentro do intervalo da trajetória estudada em animais da raça Nelore

\begin{tabular}{lcccc}
\hline Idade & $\mathrm{h}^{2}$ & $\mathrm{pa}^{2}$ & $\mathrm{pm}^{2}$ & $\mathrm{~h}^{2} \mathrm{~m}$ \\
\hline 90 & 0,256 & 0,206 & 0,155 & 0,006 \\
150 & 0,295 & 0,322 & 0,139 & 0,045 \\
210 & 0,33 & 0,343 & 0,13 & 0,051 \\
280 & 0,324 & 0,333 & 0,095 & 0,032 \\
365 & 0,336 & 0,413 & 0,067 & 0,016 \\
450 & 0,356 & 0,473 & 0,044 & 0,006 \\
550 & 0,351 & 0,525 & 0,038 & 0,004 \\
610 & 0,434 & 0,469 & 0,036 & 0,005 \\
\hline
\end{tabular}

Os resultados encontrados para a correlação genética aditiva direta foram compatíveis com os obtidos por Meyer (1998) e Santoro et al. (2005), apresentando baixas correlações entre pesos nas idades iniciais e pesos nas idades finais (Fig.3), porém com os pesos altamente correlacionados em idades próximas.

As correlações genéticas estimadas entre os pesos da desmama aos 610 dias de idade foram altas e positivas, indicando que grande parte dos genes que influenciam o aumento desses pesos são os mesmos, ou seja, a seleção para pesos em idades mais jovens pode alterar o peso em idades avançadas na mesma direção, corroborando Santos et al. (2005), Boligon et al. (2008) e Bertipaglia (2013). Esses resultados são reafirmados pela observação dos autovalores da matriz núcleo da função de covariância do efeito genético aditivo, que indicou maior peso para o primeiro autovalor $(84,44 \%)$ sugerindo, da mesma forma, que a seleção feita para aumento de peso em quaisquer idades equivale a elevar o peso nas demais idades, fato semelhante ao observado por Meyer (2003), Santoro et al. (2005) e Boligon et al. (2009).

De forma geral, verificam-se maiores associações genéticas entre idades mais próximas, enquanto o peso ao nascimento apresentou-se pouco correlacionado com os pesos nas demais idades, o que é interessante do ponto de vista econômico, visto que a seleção para o aumento do peso à desmama, ao ano ou ao sobreano não conduziria a respostas correlacionadas desfavoráveis com relação à seleção de reprodutores no que se refere à facilidade de partos. 


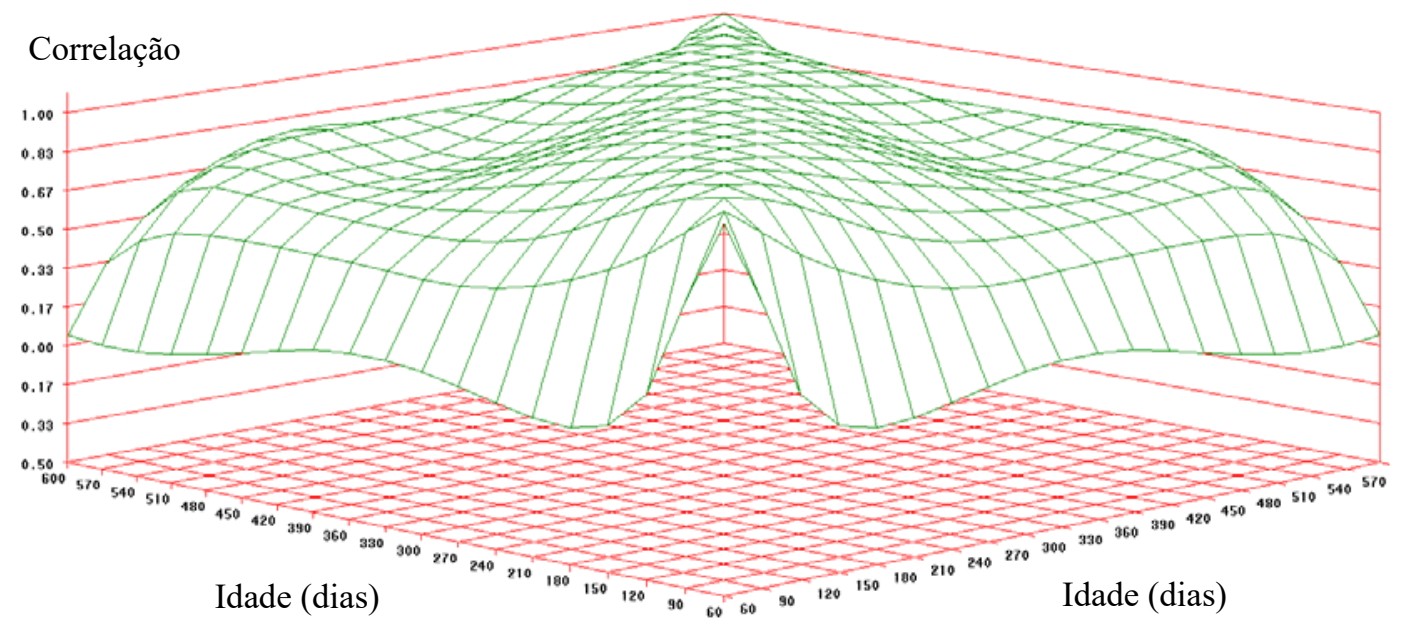

Figura 3. Correlações genéticas para o peso corporal em diferentes idades ao longo da trajetória de crescimento em animais da raça Nelore.

\section{CONCLUSÕES}

O modelo de regressão aleatória mais adequado para descrever as variâncias dos efeitos aleatórios genético aditivo direto, de ambiente permanente do animal e materno, além do efeito genético materno do peso corporal dos animais avaliados foi o que empregou função de covariância com polinômios de quarta ordem para descrição da variabilidade de todos os efeitos e duas classes de variância residual. Existe variabilidade genética expressiva nos rebanhos avaliados, o que implica ganhos genéticos satisfatórios por meio da seleção. As estimativas de herdabilidade e de correlações genéticas obtidas para o peso corporal indicam que a seleção dos animais com maiores pesos deve ser realizada considerando pesos após a desmama, uma vez que, nesse período, será possível melhor identificação dos animais geneticamente superiores.

\section{REFERÊNCIAS}

ALBUQUERQUE, L.G.; MEYER, K. Estimates of covariance functions for growth from birth to 630 days of age in Nelore cattle. J. Anim. Sci., v.79, p.2776-2789, 2001.

ARANGO, J.A.; CUNDIFF, L.V.; VAN VLECK, L.D. Covariance functions and random regression models for cow weight in beef cattle. J. Anim. Sci., v.82, p.54-67, 2004.
BALDI, F.; ALBUQUERQUE, L.G.; ALENCAR, M.M. Random regression models on Legendre polynomials to estimate genetic parameters for weights from birth to adult age in Canchim cattle. J. Anim. Breed.Gen., v.127, p.289-299, 2010.

BERTIPAGLIA, T.S. Estimativas de parâmetros genéticos para pesos do nascimento aos dois anos de idade para bovinos da raça Brahman utilizando modelos de regressão aleatória. 2013. 51f. Dissertação (Mestrado em Genética e Melhoramento Animal) - Faculdade de Ciências Agrárias e Veterinárias, Universidade Estadual Paulista, Jaboticabal, SP.

BOLIGON, A.A.; ALBUQUERQUE, L.G.; RORATO, P.N. et al. Associações genéticas entre pesos e características reprodutivas em rebanhos da raça Nelore. Rev. Bras. Zootec., v.37, p.596-601, 2008.

BOLIGON, A.A.; MERCADANTE, M.E.Z.; BALDI, F. et al. Multi-trait and random regression mature weight heritability and breeding value estimates in Nelore cattle. S. Afr. J. Anim. Sci., v.39, p.145-148, 2009.

DIAS, L.T.; ALBUQUERQUE, L.G.; TONHATI, H. Estimação de parâmetros genéticos para peso em diferentes idades para animais da raça Tabapuã. Rev. Bras. Zootec., v.35, p.1915-1925, 2006. 


\section{Araújo et al.}

HUISMAN, A.E.; VEERKAMP, R.F.; ARENDONK, J.A.M. Genetics parameters for various rando $\mathrm{m}$ regression models to describe the weight data of pigs. J. Anim. Sci., v.80, p.575-582, 2002.

KIRKPATRICK, M., LOFSVOLD, D., BULMER, M. Analysis of the inheritance, selection and evolution of growth trajectories. Genetics, v.124, p.979-993, 1990.

MEYER, K. Estimating covariance functions for longitudinal data using random regression model. Gen. Sel. Evol., v.30, p.221-240, 1998.

MEYER, K. Estimates of genetic and phenotypic covariance functions for post weaning growth and mature weight of beef cows. J. Anim. Breed. Gen., v.116, p.181-205, 1999.

MEYER, K. First estimates of covariance functions for lifetime growth of Angus cattle. Proc. Assoc. Adv. Anim. Breed. Gen., v.15, p.395-398, 2003.

MEYER, K. Estimates of genetic covariance functions for growth of Angus cattle. J. Anim. Breed. Gen., v.122, p.73-85, 2005.

MEYER, K. "WOMBAT" - Digging deep for quantitative genetic analyses by restricted maximum likelihood. In: WORLD CONGRESS ON GENETIC APPLIED TO LIVESTOCK PRODUCTION, 8., 2006, Belo Horizonte. Proceedings... Belo Horizonte, WCGALP, 2006. CD-ROM.

NEPHAWE K.A. Application of random regression models to the genetic evaluation of cow weight in Bonsmara cattle of South Africa. S. Afr. J. Anim. Sci., v.34, p.166-173, 2004.
NISHIDA, A.; AZIZ, M.A.; NISHIDA, S.; SUZUKI, K. Modeling number of services per conception of Japanese Black cattle by random regression. J. Anim. Breed. Gen., v.123, p.56-63, 2006.

NOBRE, P.R.C.; MISZTAL, I.; TSURUTA, S. et al. Analyses of growth curves of Nellore cattle by multiple-trait and random regression models. J. Anim. Sci., v.81, p.918-926, 2003.

SANTORO, K.R.; BARBOSA, S.B.P.; SANTOS, E.S. et al. Uso de funções de covariância na descrição do crescimento de bovinos Nelore criados no Estado de Pernambuco. Rev. Bras. Zootec., v.34, p.22902297, 2005.

SOUSA JÚNIOR, S.C.; OLIVEIRA, S.M.P.; ALBUQUERQUE, L.G. et al. Estimação de funções de covariância para características de crescimento da raça Tabapuã utilizando modelos de regressão aleatória, Rev. Bras. Zootec., v.39, p.1037-1045, 2010.

TORAL, F. B.; ALENCAR, M. A.; FREITAS, A. R. Estruturas de variância residual para estimação de funções de covariância para o peso de bovinos da raça Canchim. Rev. Bras. Zootec., v.38, p.2152-2160, 2009.

WOLFINGER, R. Covariance structure selection in general mixed models. Commun. Stat., v.22, p.1079-1106, 1993. 